\title{
Fenología y potencial ornamental de Euphorbia fulgens Karw. ex Klotzsch en México
}

\author{
Phenology and ornamental potential of Euphorbia fulgens Karw. ex Klotzsch in Mexico \\ Mónica Pérez-Nicolás ${ }^{1}$ (D) , María Teresa Colinas-León ${ }^{1 *}$ (D) , Iran Alia-Tejacal ${ }^{2}$ (D) \\ Margarita Gisela Peña-Ortega ${ }^{1}$
}

${ }^{1}$ Departamento de Fitotecnia, Universidad Autónoma Chapingo, Km 38.5 Carr. México-Texcoco, Chapingo, 56230, Estado de México, México.

${ }^{2}$ Facultad de Ciencias Agropecuarias, Universidad Autónoma del Estado de Morelos, Av. Universidad 1001, Cuernavaca, 62209, Morelos, México.

*Autor para correspondencia: lozcol@gmail.com

\section{Fecha de recepción:}

18 de septiembre de 2020

Fecha de aceptación:

22 de mayo de 2021

Disponible en línea:

24 de agosto de 2021

Este es un artículo en acceso abierto que se distribuye de acuerdo a los términos de la licencia Creative Commons.

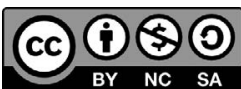

Reconocimiento-

NoComercial-

CompartirIgual 4.0

Internacional

\section{RESUMEN}

Euphorbia fulgens Karw. ex Klotzsch es una especie cultivada como planta de ornato principalmente en Europa. En México no se cultiva, a pesar de ser nativa y microendémica. El objetivo fue describir su fenología bajo condiciones de invernadero y evaluar su potencial ornamental. Se sembraron 50 semillas obtenidas de tres poblaciones silvestres; se seleccionaron aleatoriamente 30 individuos, se utilizó la escala BBCH (Biologische Bundesanstalt, Bundessortenamt und Chemische Industrie) para registrar las etapas fenológicas y se determinó el tiempo térmico. El potencial se definió con base en valores estéticos y su probable adaptación a cultivo. Se registraron siete estados fenológicos principales; las etapas de floración y fructificación se traslapan durante el periodo invierno-primavera. La etapa de plena floración se observó a los 239 DDS y fueron necesarias 3,680 ${ }^{\circ} \mathrm{C} \mathrm{día}^{-1}$. Los valores estéticos fueron ramas arqueadas con inflorescencias atractivas de colores naranja a rojo brillante y fácil adaptación a cultivo. La especie tuvo un ciclo con floración de calidad y una vida de más de 15 días.

\section{PALABRAS CLAVE}

$\mathrm{BBCH}$, flor de corte, unidades calor.

\section{ABSTRACT}

Euphorbia fulgens Karw. ex Klotzsch is a species cultivated as an ornamental plant in Europe. Despite being a native and microendemic species in Mexico, it is not cultivated in this country. The objectives were to evaluate its ornamental potential and describe its phenology under greenhouse conditions. Fifty seeds of three wild populations were sown randomly obtaining 30 individuals. The BBCH (Biologische Bundesanstalt, Bundessortenamt und Chemische Industrie) scale was used to record the phenological stages, and the thermal time was determined as well. Its potential was defined based on aesthetic values and its adaptation for cultivation. Seven main phenological stages were recorded, with the flowering and fruiting stages overlapping during the winter-spring period. The full flowering stage took place 239 days after sowing, accumulating $3,680^{\circ} \mathrm{C}$ day $^{-1}$. The aesthetic values were arched branches with attractive orange to bright red inflorescences, and easy adaptation to cultivation. The species showed a quality flowering cycle and shelf life of more than fifteen days.

\section{KEYWORDS}

$\mathrm{BBCH}$, cut flower, heat units. 


\section{INT RODUCCIÓN}

Las plantas ornamentales se utilizan con propósitos decorativos y culturales. Se pueden clasificar por su forma de uso en plantas de exterior y de interior, que pueden ser en maceta o flores de corte. También se clasifican de acuerdo con la parte decorativa, que puede ser toda la planta (arquitectura) o por follaje, flores y frutos, así como por color, olor, formas, texturas o una combinación de ellos (Li y Zhou 2005; Hurrel 2016). Son un recurso que no ha recibido la atención necesaria y su producción en México se enfoca principalmente en variedades originadas en el extranjero de plantas como crisantemo, rosa, gladiola, clavel, nochebuena y gerbera (SIAP 2018), a pesar de contar con un extenso número de especies con potencial para ser utilizadas como plantas de ornato (Borys y Leszczyñska 1992; Corona y Chimal 2006; Munguía-Lino et al. 2010), amplia diversidad de climas y suelos, mano de obra suficiente y cercanía con Estados Unidos, uno de los consumidores más importantes del mundo (Tejeda-Sartorius y Arévalo-Galarza 2012).

Se han llevado a cabo estudios sobre el potencial ornamental de grupos de plantas como helechos (Díaz-Barriga 1995; Gaspar-Génico 2002) y plantas acuáticas (Bonilla-Barbosa 1994); de familias como Orchidaceae (López et al. 1995), Acanthaceae (Ramírez-Hernández 2005) y Bromeliaceae (Arellano-Mijangos 2002); de géneros como Pinguicula (Zamudio 1995) y Begonia (Corona et al. 1995), y de especies como Sprekelia formosissima (L.) Herb. (Torres-Navarro 2000). También se han enlistado especies en Morelos (Rendón y Fernández 2007), Querétaro (Pérez-Nicolás y Fernández 2007) y el Estado de México (Guadarrama-Martínez et al. 2012). En los trabajos anteriores, el potencial es definido por características o valores estéticos que presentan las especies; no obstante, Pérez-Nicolás et al. (2020) proponen que en especies silvestres se debe incluir una evaluación del comportamiento bajo un primer ciclo de cultivo.

Euphorbia fulgens Karw. ex Klotzsch es una planta con potencial ornamental, pues se comercializa principalmente como flor de corte en Holanda, donde se conoce con el nombre común "scarlet plume" (pluma escarlata); se ofrecen diversas variedades que presentan inflorescencias de diferentes colores: anaranjado, rosa, rojo, blanco, salmón, durazno y amarillo, así como hojas de color verde, rojo oscuro o morado (Flower Nursery De Googh 2018). También se comercializa en Estados Unidos (Calix Flowers 2020; Royer's Flowers \& Gifts 2020), Australia (Weslor Flowers Plant Nusery 2020), Canadá (Pink Ontario 2020), India (Nursery Pionner 2020) e Inglaterra (Triangle Nursery Ltd. 2010). Son pocas las investigaciones efectuadas sobre esta especie: se ha estudiado la estivación del ciatio (Schoute 1937); se ha evaluado el efecto de factores como la temperatura y humedad en la inducción y floración (Rünger y Albert 1975); se han documentado técnicas para su cultivo in vitro (Zhang et al. 1987), así como tratamientos postcosecha para evitar el amarillamiento y abscisión de hojas (Van Leeuwen 1986; Jiang et al. 2009).

Debido a que se desconocen diversos aspectos sobre el cultivo de E. fulgens, es necesario describir las etapas fenológicas, pues su determinación resulta fundamental y útil en el manejo agronómico de la especie. La escala $\mathrm{BBCH}$ (Biologische Bundesanstalt, Bundessortenamt und Chemische Industrie) es un sistema de codificación decimal utilizado para uniformar las etapas fenológicas similares en las monocotiledóneas y dicotiledóneas (Enz y Dachler 1998; Meier et al. 2009); se ha utilizado en Dimocarpus longan Lour. (Pham et al. 2015), Amaranthus cruentus L., A. hybridus L., A. hypochondriacus L. (Martínez-Núñez et al. 2019), Salvia hispanica L. (Pérez et al. 2019), Setaria viridis (L.) P. Beauv. (Junqueira et al. 2020), entre otras.

La determinación de las etapas fenológicas en función del tiempo térmico, tiempo fisiológico, grados día de crecimiento (GDC) o unidades calor (UC) es más adecuada porque la temperatura posee una influencia directa sobre el crecimiento y desarrollo vegetal (Bonhomme 2000; Salazar-Gutiérrez et al. 2013). Uno de los métodos más utilizados para el cálculo de unidades calor es el presentado por Snyder (1985), que se basa en la acumulación de una temperatura media diaria por arriba de la temperatura base $(\mathrm{Tb})$ de la especie en cuestión. Así, cada especie vegetal, e incluso cultivares y variedades, presentan requerimientos térmicos diferentes (Sikder 2009). Son pocos los trabajos efectuados en plantas ornamentales en donde se han definido las unidades calor necesarias para llegar a determinada etapa fenológica, por ejemplo, Gladiolus grandiflorus Andrews (Ocampo-Juárez et al. 2012; González-Pérez et al. 2011), en Dianthus caryophyllus 
L. (López et al. 2010) y Euphorbia pulcherrima Karw. ex Klotzsch (Pérez-López et al. 2005).

Por lo anterior, el presente trabajo tuvo como objetivo evaluar el potencial ornamental en México de E. fulgens, registrar su fenología bajo condiciones de invernadero y determinar las unidades calor necesarias para las etapas fenológicas: emergencia, primer par de hojas opuestas, botones visibles y plena floración.

\section{Materiales y Métodos}

\section{Material vegetal y establecimiento bajo invernadero}

En abril de 2018, se colectaron frutos de E. fulgens en tres localidades del estado de Oaxaca. El experimento se llevó a cabo en un invernadero de cristal ubicado en la Universidad Autónoma Chapingo, la cual se localiza a $19^{\circ} 20^{\prime}$ latitud norte y $98^{\circ} 53^{\prime}$ longitud oeste, a 2,240 $\mathrm{m}$ de altitud. La temperatura y humedad relativa se registró con un data logger $\mathrm{HOBO}{ }^{\circledR}$ U12-012 (Onset Computer Corporation, Massachusetts, Estados Unidos); la temperatura media en ese periodo varió de 16 a $24{ }^{\circ} \mathrm{C}$ y la humedad relativa de 38 a 68 por ciento.

De cada sitio se seleccionaron 50 semillas, las cuales se sembraron el 10 de mayo del mismo año, en macetas $(7.5 \times 5.5 \times 5.1 \mathrm{~cm})$ llenadas con un sustrato de turba y perlita, en una proporción 50:50 v/v. Cuando las plántulas mostraron la cuarta hoja verdadera, se hizo el trasplante a bolsas negras de polietileno ( $25 \mathrm{x}$ $25 \mathrm{~cm}$ ), llenadas con un sustrato de tierra negra/tierra de hoja de encino/corteza de pino/fibra de coco/vermicomposta (20:30:20:20:10 v/v). Se colocaron bajo una malla sombra de polietileno a 50 por ciento. Las plantas se regaron manualmente una o dos veces por semana. Se comenzó el tutoreo cuando éstas alcanzaron $30 \mathrm{~cm}$; entonces, se colocaron varillas de madera de $0.7 \mathrm{~mm}$ de diámetro y $90 \mathrm{~cm}$ de longitud.

En la primera etapa, se utilizó el fertilizante inicial Ultrasol@ inicial N15-P30-K15+M.E. AQTEX $0.5 \mathrm{~g}$ $\mathrm{L}^{-1}, \mathrm{y}$, en la segunda, un fertilizante multipropósito N18-P18-K18 + M.E. AQTEX, 1 g L$^{-1}$. Para prevenir la aparición de hongos se aplicó benomilo (Promyl ${ }^{\circledR}, 1.5$ g L-1); asimismo, para la prevención de mosca negra se usó bifentrina (Talstar®, $1.5 \mathrm{~mL} \mathrm{~L}^{-1}$ ), alternando con cipermetrina (Siroco® $1.5 \mathrm{~mL} \mathrm{~L}^{-1}$ ), y se colocaron trampas cromáticas amarillas y azules. Se presentó araña roja
(Tetranychus urticae Koch), la cual se controló con abamectina (Agrimec ${ }^{\circledR} 1.5 \mathrm{~mL} \mathrm{~L}-1$ ) y Bacillus thuringiensis Berliner. El control de malezas se llevó a cabo de manera manual.

\section{Fenología}

Para registrar las etapas fenológicas, se seleccionaron aleatoriamente 10 individuos de cada sitio, por lo que, en total, se evaluaron 30 plantas usando la escala $\mathrm{BBCH}$ (Enz y Dachler, 1998; Pham et al. 2015), para especies monocotiledóneas y dicotiledóneas. La escala usa un código decimal que se aplica a las mismas etapas de crecimiento de diferentes especies de plantas. El ciclo de crecimiento completo de una planta se divide en 10 etapas principales, codificadas de 0 a 9, y éstas se subdividen en etapas secundarias que también se enumeraron del 0 al 9.

La acumulación de unidades térmicas $\left(\mathrm{UC},{ }^{\circ} \mathrm{C}\right.$ día) se calculó mediante el método residual presentado por Snyder (1985), que utiliza el modelo: $\mathrm{UC}=($ Tmax + Tmin/2) - Tb; donde Tmax = Temperatura máxima diaria $\left({ }^{\circ} \mathrm{C}\right)$; Tmin $=$ Temperatura mínima diaria $\left({ }^{\circ} \mathrm{C}\right) \mathrm{y}$ $\mathrm{Tb}=$ Temperatura base $\left(5^{\circ} \mathrm{C}\right.$ para Euphorbia pulcherrima Willd ex. Klotzsch, de acuerdo con Pérez-López et al. (2005).

\section{Potencial ornamental en México}

Se definió el potencial ornamental de la especie mediante el análisis de los valores estéticos (Pérez-Nicolás y Fernández 2007, Rendón y Fernández 2007) que presenta, es decir, a partir de aspectos decorativos: arquitectura de la planta, flores, frutos, color, olor, formas, texturas o la combinación de varios elementos (Li y Zhou 2005; Hurrel 2016). Por último, se evaluó en qué fase del proceso de investigación de nuevos cultivos se encuentra. Las tres fases son: 1) propagación y establecimiento bajo invernadero, 2) producción y 3) comercialización (Pérez-Nicolás et al. 2020).

\section{RESUltados Y Discusión}

\section{Etapas fenológicas}


Euphorbia fulgens bajo cultivo en invernadero presentó siete etapas fenológicas; de acuerdo con el código $\mathrm{BBCH}$, corresponden a una fase vegetativa (Cuadro 1) y a una etapa reproductiva (Cuadro 2). En otros estudios se han registrado de siete a nueve etapas fenológicas principales de acuerdo con $\mathrm{BBCH}$; por ejemplo, en Amaranthus spp., que es una herbácea, se registraron nueve estados fenológicos desde semilla hasta senescencia (Martínez-Núñez et al. 2019), y en Dimocarpus longan, que es un árbol, se registraron siete etapas fenológicas desde yema vegetativa hasta maduración del fruto (Pham et al. 2015).

La germinación fue epigea y la emergencia escalonada (09) durante siete semanas. En las obtenidas del sitio uno, la emergencia ocurrió de los 13 días después de la siembra (DDS) hasta los 39 DDS; en las del sitio dos, fue de los 15 DDS a los 40 DDS, y las del sitio tres, del día 15 DDS hasta el día 45 DDS (Figura 1). El hipocótilo emergió curvado con los cotiledones todavía envueltos en la testa; un día después se enderezaron y elongaron, se desprendió la testa y hubo apertura de cotiledones; en algunas plantas, no hubo desprendimiento de la testa y los cotiledones quedaron encerrados por la misma. En los dos días siguientes, los cotiledones se desarrollaron completamente (10). Tres días después aparecieron las primeras hojas opuestas (11); después de otros tres o cuatro días, la primera hoja alterna (12), y tres o cuatro días después, la tercera hoja verdadera (13).

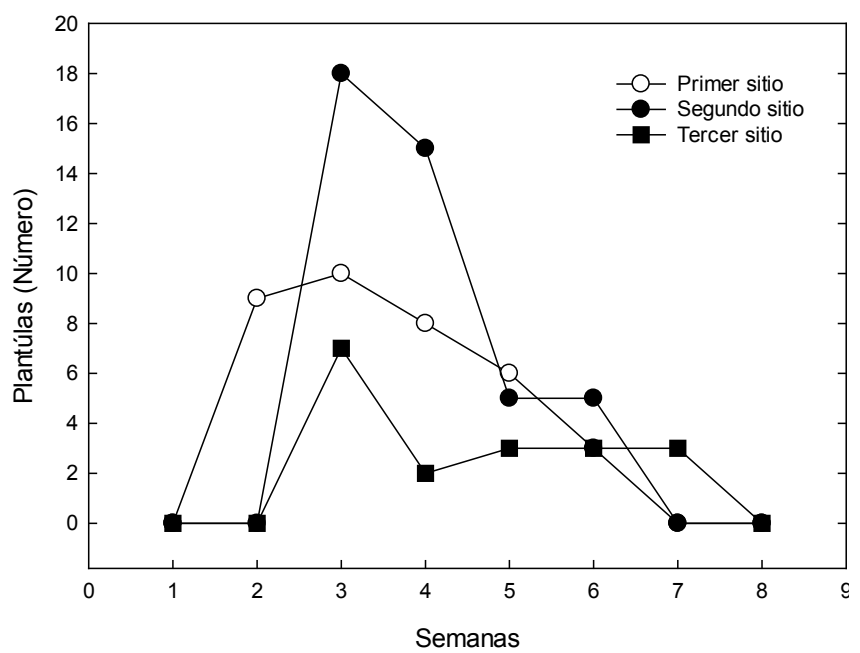

Figura 1. Emergencia de plántulas de Euphorbia fulgens provenientes de semillas colectadas en poblaciones silvestres ubicadas en Oaxaca.
En los meses de junio, julio y principios de agosto las plantas tuvieron 9 o más hojas verdaderas (19); en agosto, septiembre y octubre, el crecimiento del tallo fue paralelo al desarrollo de las hojas (Figura 2). A finales de septiembre, se observaron las primeras yemas florales; en octubre, los primeros botones y 50 por ciento de los individuos tuvo botones florales; en noviembre (51), posteriormente, emergen los apéndices petalíferos (59) y días después las estructuras reproductivas: primero las flores masculinas reducidas a estambres y después una sola flor femenina (estilo y estigma), que integran el ciatio característico en el género Euphorbia (60); cabe mencionar que en la misma planta podemos encontrar ciatios masculinos, femeninos y hermafroditas. La plena floración se observó los últimos días de diciembre y los primeros de enero (etapa 65). Los frutos alcanzaron su tamaño específico en enero y febrero (71), y se cosecharon a finales de febrero y principios de marzo (89) (Figura 2).
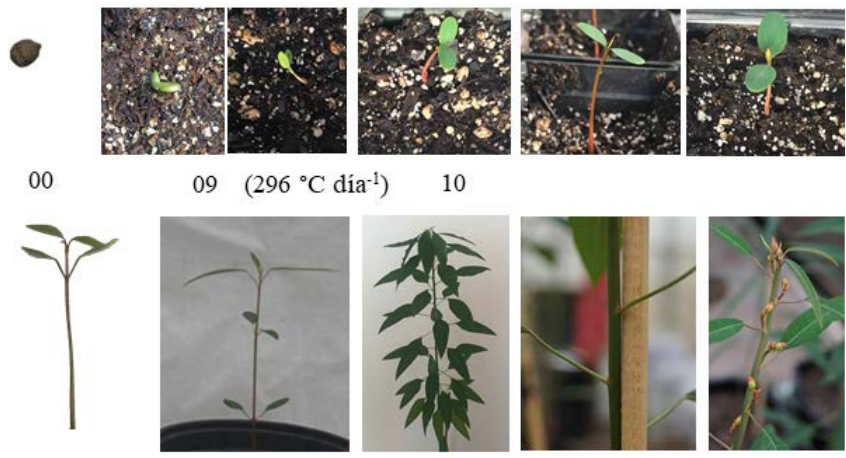

$09\left(296{ }^{\circ} \mathrm{C} \mathrm{día}^{-1}\right) \quad 10$
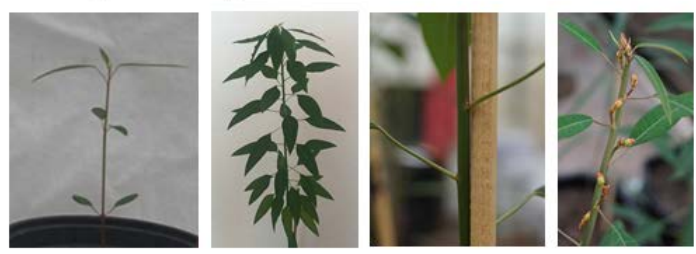

$11\left(388^{\circ} \mathrm{C} \mathrm{dia}{ }^{-1}\right) \quad 12$
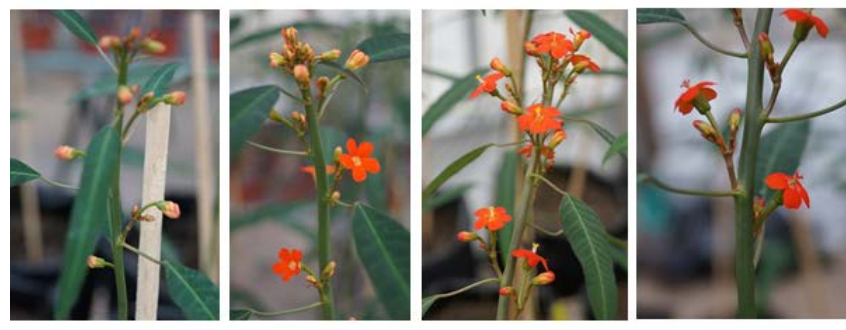

$51\left(2,960{ }^{\circ} \mathrm{C} \mathrm{día}{ }^{-1}\right) \quad 59$

60
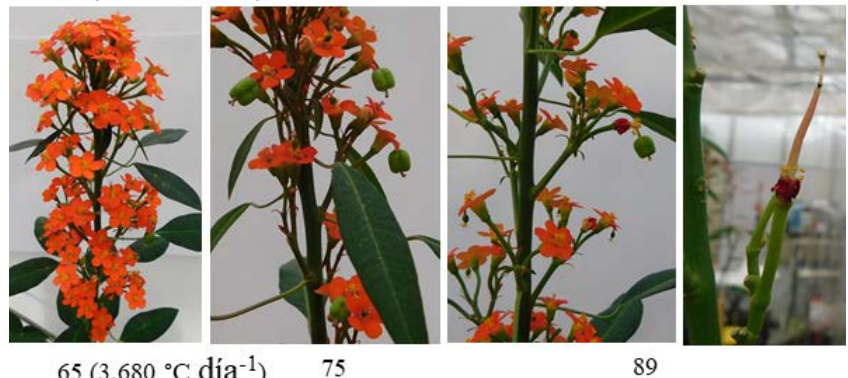

89

Figura 2. Etapas fenológicas en Euphorbia fulgens cultivada bajo invernadero. 
Cuadro 1. Estados fenológicos determinados en fase vegetativa de Euphorbia fulgens de acuerdo con la escala BBCH con base en

\begin{tabular}{|c|c|c|c|}
\hline $\begin{array}{l}\text { Estadios principales } \\
\text { (código } \mathrm{BBCH} \text { ) }\end{array}$ & Descripción & $\begin{array}{l}\text { Código } \\
\text { BBCH }\end{array}$ & DDS \\
\hline \multirow{2}{*}{$\begin{array}{l}\text { 0: Germinación } \\
\text { Siembra } 10 \text { mayo }\end{array}$} & Semilla seca & 00 & 0 \\
\hline & $\begin{array}{l}\text { Emergencia: los cotiledones salen a través de la superficie } \\
\text { del suelo } \\
\text { Escalonada del } 23 \text { mayo hasta el } 24 \text { de junio }\end{array}$ & 09 & 16 \\
\hline \multirow{5}{*}{$\begin{array}{l}\text { 1: Desarrollo de hojas (tallo } \\
\text { principal) } \\
\text { Mayo-junio }\end{array}$} & Cotiledones completamente desarrollados & 10 & 18 \\
\hline & Desarrollo del primer par de hojas verdaderas (opuestas) & 11 & 21 \\
\hline & Desarrollo de la segunda hoja verdadera (alterna) & 12 & 24 \\
\hline & Desarrollo de la tercera hoja verdadera (alterna) & 13 & 28 \\
\hline & Desarrollo de 9 o más hojas verdaderas & 19 & 90 \\
\hline $\begin{array}{l}\text { 3: Elongación del tallo } \\
\text { Julio-octubre }\end{array}$ & El crecimiento del tallo es paralelo al desarrollo de las hojas & -- & -- \\
\hline
\end{tabular}

DDS: Días después de la siembra.

Cuadro 2. Estados fenológicos en fase reproductiva de Euphorbia fulgens de acuerdo con la escala BBCH y con base en $50 \%$ de los individuos.

\begin{tabular}{|c|c|c|c|}
\hline $\begin{array}{l}\text { Estadios principales } \\
\text { (código } \mathrm{BBCH} \text { ) }\end{array}$ & Descripción & $\begin{array}{l}\text { Código } \\
\text { BBCH }\end{array}$ & DDS \\
\hline \multirow{2}{*}{$\begin{array}{l}\text { 5: Aparición de órganos } \\
\text { florales } \\
\text { Octubre-noviembre }\end{array}$} & Botones florales visibles & 51 & 178 \\
\hline & Primeros apéndices petalíferos visibles & 59 & 195 \\
\hline \multirow{3}{*}{$\begin{array}{l}\text { 6: Floración } \\
\text { Diciembre-enero }\end{array}$} & Primeros ciatios abiertos (se observan estambres y estigmas) & 60 & 209 \\
\hline & $\begin{array}{l}30 \% \text { de ciatios visibles: ciatios hermafroditas, masculinos y } \\
\text { femeninos }\end{array}$ & 63 & 226 \\
\hline & $\begin{array}{l}\text { Plena floración: } 50 \% \text { de las flores abiertas, los primeros } \\
\text { apéndices petalíferos se secan }\end{array}$ & 65 & 239 \\
\hline \multirow{3}{*}{$\begin{array}{l}\text { 7: Formación del fruto } \\
\text { Enero-febrero }\end{array}$} & Primeros frutos visibles & 70 & 255 \\
\hline & $30 \%$ de los frutos alcanza el tamaño específico de su especie & 71 & 268 \\
\hline & $50 \%$ de los frutos alcanza el tamaño específico de su especie & 75 & 277 \\
\hline \multirow{2}{*}{$\begin{array}{l}\text { 8: Maduración de frutos y } \\
\text { semillas } \\
\text { Febrero-marzo }\end{array}$} & Comienzo de la maduración & 81 & -- \\
\hline & $\begin{array}{l}\text { Maduración plena. Los frutos se desprenden con relativa } \\
\text { facilidad }\end{array}$ & 89 & 306 \\
\hline
\end{tabular}

DDS: Días después de la siembra.

Cabe aclarar que en un mismo individuo se pueden encontrar diferentes etapas de desarrollo, es decir, botones, ciatios únicamente con apéndices petalíferos, ciatios masculinos, ciatios femeninos, ciatios hermafroditas y frutos. Aunque se observó que los botones generalmente no se desarrollan si 30 por ciento de los frutos ya se encuentran totalmente desarrollados, lo atractivo de las plantas son los apéndices petalíferos que se asemejan a los pétalos de una flor, y éstos se observan desde finales de noviembre hasta principios de febrero. 
La emergencia de las plántulas de E. fulgens requirió 296 ${ }^{\circ} \mathrm{C}$ día $^{-1}$, el desarrollo del primer par de hojas opuestas $388{ }^{\circ} \mathrm{C}$ día ${ }^{-1}$, el desarrollo de la segunda y tercera hoja verdaderas 439 y $508{ }^{\circ} \mathrm{C}$ día ${ }^{-1}$, respectivamente. En cuanto a la fase reproductiva, los botones fueron visibles a los 2,960 ${ }^{\circ} \mathrm{C}$ día ${ }^{-1} \mathrm{y}$ la plena floración ocurrió a los 3,680 ${ }^{\circ} \mathrm{C}$ día $^{-1}$. El ciclo de desarrollo fue largo: la siembra se llevó a cabo el 10 de mayo, y la mayoría de los individuos estuvo en plena floración a principios de enero, es decir, ocho meses después; esto puede deberse a que se trabajó con semillas provenientes de poblaciones silvestres que, en condiciones naturales, presenta estados fenológicos similares. Las unidades de calor requeridas fueron elevadas, en comparación con especies cultivadas, cuyos ciclos de cultivo son más cortos y requieren menor cantidad de unidades de calor para llegar a la etapa de interés agrícola. En nochebuena se evaluó el ciclo desde el despunte hasta la antesis y los grados días de desarrollo (ggd) acumulados en 147 días fueron de $2,270{ }^{\circ} \mathrm{C} \mathrm{d}^{-1}$ por el método residual, y por el método Manrique y Hodges fueron de $1,350{ }^{\circ} \mathrm{C}$ $\mathrm{d}^{-1}$ (Pérez-López et al. 2005). En Gladiolus grandiflorus L., la apertura de la primera flor basal fue a los 82 DDS y la madurez fisiológica del cormo y hasta cosecha se presentó a los $161 \mathrm{DDS}$; se registraron $2,630{ }^{\circ} \mathrm{C}$ días que corresponden a la fase vegetativa y reproductiva; en esta última, se registraron los valores más altos de unidades de calor (Ocampo-Juárez et al. 2012), al igual que en E. fulgens.

En Dianthus caryophyllus, a partir del pinch y hasta la aparición del botón floral, se acumularon 1,363.2 ggd y el pico de cosecha se alcanzó a los 2,226.5 ggd (López et al. 2010). Las unidades de calor requeridas pueden variar a nivel de variedad y en diferentes localidades; en Rosa sp., las unidades de calor requeridas desde el pinch hasta corte de flor dependieron de la localidad y tipo de variedad, en 66 días fueron 900 unidades calor, mientras que en 87 días fueron 1,022 (De la Rosa 2013). En este trabajo, se analizó el ciclo completo de $E$. fulgens, desde semilla hasta fructificación, debido a que no se cultiva en nuestro país; sin embargo, se sugiere realizar determinaciones en plantas provenientes de esquejes y establecer el cultivo en zonas con climas parecidos a donde habitan de manera silvestre y llevar a cabo manejo agronómico, enfocado en tener al menos dos ciclos de producción reduciendo el tiempo de ciclo de cultivo.

\section{Potencial ornamental en México}

Su comportamiento bajo invernadero fue óptimo, pues es una especie que se adapta a condiciones de cultivo: florece bajo condiciones de día corto, al igual que las variedades comercializadas en otros países, que se establecen bajo sombra parcial, con una duración crítica del día de 11 a 12 horas, dependiendo de la temperatura (Rünger y Albert 1975; Flower Nursery De Googh 2018; The Royal Horticultural Society 2020).

En el primer ciclo de cultivo, las plantas se establecieron en macetas, a diferencia de lo que se hace en otros países, donde se cultiva como flor de corte, dentro de invernaderos, en camas con sustratos arenosos (Flower Nursery De Googh 2018). Se partió de semillas porque en México no se cuenta con materiales mejorados; sin embargo, se recomienda propagar por esquejes, debido a que es una especie perenne que presenta brotes vegetativos. En Holanda, los cultivares se propagan por esquejes que se cortan en los meses de mayo y junio, para obtener la producción a finales de otoño y durante el invierno (Flower Nursery De Googh 2018).

Las condiciones de manejo fueron similares a las reportadas en otros países, que corresponden a un rango de temperatura para un buen desarrollo de 21 a $26^{\circ} \mathrm{C}$, el pH del suelo es de 6.5 o neutral y el riego dos veces a la semana, dejando el sustrato húmedo, no mojado, aunque depende de las condiciones donde se establezca el cultivo. Se requiere reducir el riego en otoño y regar mínimamente en invierno para un mejor rendimiento (Flower Nursery De Googh 2018; Pink Ontario 2020; The Royal Horticultural Society 2020). Para su cultivo, se sugiere usar sustratos arenosos con buena aireación y no realizar riegos abundantes.

Las plantas establecidas en México presentaron diferentes valores estéticos: uno de ellos es la arquitectura de la planta por sus ramas arqueadas, y otro es la parte que florece, compuesta por diversas cimas de ciatios con apéndices petalíferos de colores atractivos, que van del anaranjado al rojo y son brillantes (Figura 3). Se observó que las inflorescencias sin mejoramiento genético son muy parecidas a algunas variedades comercializadas en otros países (Triangle Nursery 2010; Flower Nursery De Googh 2018; Pink Ontario 2020). La especie pasó la fase I del proceso para generar 
nuevos cultivos, la cual consiste en tener un ciclo bajo invernadero con floración, en el que conservó su calidad y tuvo una vida de más de 15 días. Se mantuvo en etapa de floración aproximadamente 4 meses; en una misma rama, se encontraron diferentes estadios de desarrollo de las flores y frutos. Sólo se presentó la plaga de la araña roja y no fue susceptible a la mosca blanca, la cual representa un problema sanitario en cultivos de nochebuena, donde se presenta en alta frecuencia y es difícil de erradicar.

Es una especie que puede ser utilizada como flor de corte, debido a que es perenne y es un sufrútice (parte basal lignificada); de igual manera, se adapta como planta en maceta y también puede ser utilizada como planta de exterior en jardines a media sombra. Se encuentra en la fase II, que consiste en la propagación para establecer la mejor técnica de cultivo, por lo que está en evaluación la propagación mediante esquejes y se llevan a cabo experimentos enfocados a evaluar factores que determinarán su manejo agronómico.
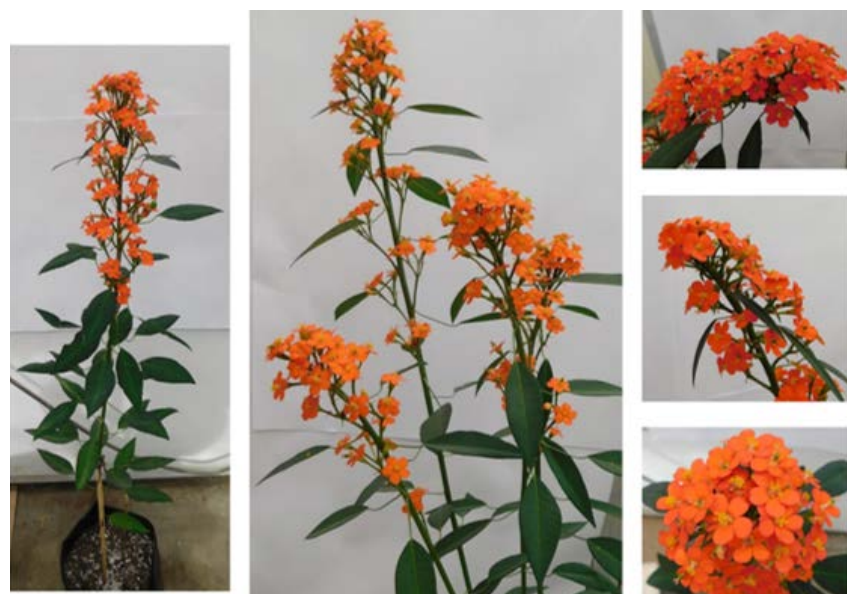

Figura 3. Valores estéticos en Euphorbia fulgens.

\section{Conclusiones}

Euphorbia fulgens presentó siete etapas fenológicas bajo cultivo en invernadero, las cuales corresponden a una fase vegetativa y a una reproductiva. La floración y fructificación se traslapan durante el periodo de invierno y primavera. La emergencia de las plántulas ocurrió 16 DDS y requirió $296{ }^{\circ} \mathrm{C}$ día ${ }^{-1}$; el desarrollo del primer par de hojas opuestas fue después de 21 DDS, con $388^{\circ} \mathrm{C}$ día $^{-1}$; los botones fueron visibles a los 178 DDS, con $2,960{ }^{\circ} \mathrm{C}$ día $^{-1}$, y, después de 239 DDS, con $3,680{ }^{\circ} \mathrm{C}_{\text {día }}{ }^{-1}$ se registró plena floración.
Euphorbia fulgens tiene potencial para ser cultivada como ornamental, principalmente por presentar ramas arqueadas, inflorescencias atractivas, larga duración en etapa de floración y adaptación a cultivo. La especie tuvo un ciclo bajo invernadero con floración, el cual mantuvo su calidad y tuvo una vida de más de 15 días; se encuentra en la Fase II del proceso de generación de nuevos cultivos, por lo que se sugiere llevar a cabo evaluaciones del efecto de la luz, temperatura, humedad, nutrición y fotoperiodo.

\section{Agradecimientos}

A Rene Aguilar y Claudio Flores, por su apoyo en el trabajo de campo. A Selene Sánchez, por su apoyo en la determinación de unidades calor. A Víctor Steinmann, por la revisión y sugerencias durante la realización de este estudio. En particular, agradecemos las observaciones y sugerencias hechas por los revisores anónimos. 


\section{LiterATURA CITADA}

Arellano-Mijangos JJ. 2002. Las Bromeliaceae del estado de Oaxaca, riqueza florística y potencial ornamental. Tesis de Licenciatura. Universidad Autónoma Chapingo. Chapingo, México.

Bonhomme R. 2000. Bases and limits to using 'degree. day' units. European Journal of Agronomy 13: 1-10. https://doi.org/10.1016/S1161-0301(00)00058-7

Bonilla-Barbosa JR. 1994. Plantas acuáticas ornamentales del estado de Morelos, México. Revista Chapingo Serie Horticultura 1: 79-83.

Borys M, Leszcyñska H. 1992. Reflexiones sobre el potencial ornamental de plantas en México. Manuales de Horticultura ornamental 7. Universidad Autónoma del Estado de Puebla. Puebla, México.

[Calix Flowers] Calix Flowers. The Flowers Lover's Flower, Inc. [internet]. 2020. Scarlet Plume - Euphorbia fulgens. [citado 2020 ene 15]. Disponible en: https:// www.calyxflowers.com/floral-library/scarlet-plume/

Corona V, Chimal A, Hernández GA. 1995. Las begonias ornamentales en México. Revista Chapingo Serie Horticultura 3: 71-76.

Corona V, Chimal A. 2006. Plantas mexicanas con potencial ornamental. Universidad Autónoma Metropolitana. Ciudad de México, México.

De la Rosa TM. 2013. Identificación de estados fenológicos y determinación de unidades calor en el cultivo de Rosa (Rosa $x$ hibrida) en la región florícola del Estado de México. Tesis de Licenciatura. Universidad Autónoma del Estado de México. Tenancingo, México.

Díaz-Barriga H. 1995. Las Pteridofitas del Bajío y sus posibilidades como plantas ornamentales. Revista Chapingo Serie Horticultura 3: 57-61.

Enz M, Dachler Ch. [internet]. 1998. Compendio para la identificación de los estadios fenológicos de especies mono- y dicotiledóneas cultivadas escala $\mathrm{BBCH}$ extendida. BBA, BSA, IVA, IGZ. [citado 2018 abril]. Disponible en: https://www.agro.basf.es/Documents/ es_files/pdf_1_files/services_files/descarga.pdf

Flower Nusery De Goohg. [internet]. 2018. Assorment Euphorbia fulgens. [citado 2018 feb]. Disponible en: https://www.googh.nl/euphorbia-fulgens-assortment.html

Gaspar-Génico JA. 2002. Las Pteridophyta silvestres del Estado de Tabasco y su potencial ornamental. Tesis de Licenciatura. Universidad Autónoma Chapingo. Chapingo, México.

González-Pérez E, Ayala-Garay O, Carillo-Salazara JA, García de los Santos G, Yáñez-Morales MdeJ, JuárezMúñoz J. 2011. Estudio del desarrollo, calidad de flor y dosis de fertilización en gladiolo (Gladiolus grandiflorus Hort.). Revista Fitotecnia Mexicana 34: 277-283.

Guadarrama-Martínez N, Rubí-Arriaga M, GonzálezHuerta A, Vázquez-García LM, Martínez-De la Cruz I, López-Sandoval JA, Hernández-Flores GV. 2012. Inventario de árboles y arbustos con potencial ornamental en el sureste del Estado de México. PYTHON, Revista Internacional de Botánica Experimental 81: 221-228.

Hurrel JA. 2016. Ornamental plants. En: Albuquerque UP, Alves R, editores. Introduction to Ethnobiology. Cham, Springer International Publishing. P. 171-176.

Jiang CZ, Wu L, Macnish AJ, King A, Yi M, Reid MS. 2009. Thidiazuron, a non-metabolized cytokinin, shows promise in extending the life of potted plants. Acta Horticulturae 847: 59-65. https://doi.org/10.17660/ ActaHortic.2009.847.6

Junqueira NEG, Bezaerra ACM, Cattem MVO, Medicin LO, Alves-Ferreira M, Macrae A, Ortiz-Silva B, Reinert F. 2020. Phenology of the genetic model Setaria viridis (Poaceae) according to the BBCH-scale of development. Botanical Journal of the Linnean Society 192: 224-241. https://doi.org/10.1093/botlinnean/boz070

Li XX, Zhou ZK. 2005. Endemic wild ornamental plants from Northwestern Yunnan, China. HortScience 40: 1612-1619. https://doi.org/10.21273/HORTSCI.40.6.1612 López A, Sosa C, Mejía J. 1995. Plantas del sureste de México con potencial ornamental: orquídeas. Revista Chapingo Serie Horticultura 3: 45-56.

López MA, Chaves B, Flórez RV, Salazar MR. 2010. Modelo de aparición de nudos en clavel (Dianthus caryophyllus L.) cv. Delphi cultivado en sustratos. Agronomía Colombiana 28: 47-54.

Martínez-Núñez M, Ruiz-Rivas M, Vera-Hernández PF, Bernal-Muñoz R, Luna-Suárez S, Rosas-Cárdenas FF. 2019. The phenological growth stages of different amaranth species grown in restricted spaces based in $\mathrm{BBCH}$ code. South African Journal of Botany 124: 436-443. http://doi.org/10.1016/j.sajb.2019.05.035

Meier U, Bleiholder H, Buhr L, Feller C, Hack H, Heß M, Lancashire PD, Schnock U, Stauß R, van den Boom T, Weber E, Zwerger P. 2009. The BBCH system to coding 
the phenological growth stages of plants - history and publications. Journal für Kulturpflanzen 61: 41-52.

Munguía-Lino G, Vázquez-García LM, López-Sandoval JA. 2010. Plantas silvestres ornamentales comercializadas en los mercados de la flor de Tenancingo y Jamaica, México. Polibotánica 29: 281-308.

Nursery Pionner. [internet]. 2020. Euphorbia fulgens (scarlet plume). [citado 2020 ene 15]. Disponible en: https:// www.nurserypioneer.com/product/euphorbia-fulgans/

Ocampo-Juárez H, Escalante-Estrada JAS, RodríguezGonzález MT, Landeros-Sánchez F, Escalante-Estrada LE. 2012. Producción de gladiolo en función del nitrógeno fósforo y potasio. Terra Latinoamericana 30: 239-248.

Pérez J, Curti RN, Acreche MM. 2019. Phenological growth stages in chia (Salvia hispanica L.) according to the BBCH scale. Scientia Horticulturae 255: 292-297. http:// doi.org/10.1016/j.scienta.2019.05.043

Pérez-López A, Carrillo-Salazar JA, Colinas-León MT, Sandoval-Villa M. 2005. Regulación del crecimiento de nochebuena (Euphorbia pulcherrima Willd ex. Klotzsch) con etefón. Agrociencia 39: 639-646.

Pérez-Nicolás M, Fernández N. 2007. Plantas del estado de Querétaro, México con potencial para uso ornamental. Polibotánica 24: 83-115.

Pérez-Nicolás $\mathrm{M}$, Colinas-Léon MT, Gómez-Alanis MC, Flores-Espinosa C, Alia-Tejacal I, Peña-Ortega MG. 2020. Distribución geográfica de especies de Euphorbia sect. Poinsettia con posible uso ornamental en México. Polibotánica 50: 165-189. http://doi.org/10.18387/ polibotanica.50.12

Pham VT, Herrero M, Hormaza JI. 2015. Phenological growth stages of longan (Dimocarpus longan) according to the BBCH scale. Scientia Horticulturae 189: 201-207. https://doi.org/10.1016/j.scienta.2015.03.036

Pink Ontario. [internet]. 2020. Euphorbia, scarlet plume (Euphorbia fulgens). [citado 2020 ene 15]. Disponible en: https://www.po.flowerscanadagrowers.com/ our-products/3698/euphorbia/fulgens

Ramírez-Hernández SG. 2005. Las Acanthaceae de Tabasco y su potencial como plantas ornamentales. Tesis de Licenciatura. Universidad Juárez Autónoma de Tabasco. Villahermosa, México.

Rendón A, Fernández N. 2007. Plantas con potencial uso ornamental del estado de Morelos, México. Polibotánica 23: 121-165.

Royer's Flowers \& Gifts. [internet]. 2020. Floral Library- Scarlet Plume. [citado 2020 ene 15].
Disponible en: https://www.royers.com/usrf. cfm?funfacts=floraldisplaypage\&floral_id $=47$

Rünger W, Albert G. 1975. Influence of temperature, soil moisture and CCC on the flowering of Euphorbia fulgens. Scientia Horticulturae 3: 393-403. https://doi. org/10.1016/0304-4238(75)90054-0

Salazar-Gutiérrez MR, Chaves-Cordoba B, Hoogenboom G. 2013. Relationship of base temperature to development of winter wheat. International Journal of Plant Production 7: 741-762.

Schoute JC. 1937. On the aestivation in the cyathium of Euphorbia fulgens, with some general remarks on the morphological interpretation of the cyathium in general. Recueil des Travvaux Botaniques Néerlandais 34: 168-181.

[SIAP] Servicio de Información Agroalimentaria y Pesquera. [internet]. 2018. Datos abiertos. [citado 2019 ago 15]. Disponible en: http://infosiap.siap.gob.mx/gobmx/datosAbiertos_a.php

Sikder S. 2009. Accumulated heat unit and phenology of wheat cultivars as influenced by late sowing heat stress condition. Journal of Agriculture \& Rural Development 7: 57-64. https://doi.org/10.3329/jard.v7i1.4422

Snyder RL. 1985. Hand calculating degree days. Agricultural and Forest Meterology 35: 353-358. https:// doi.org/10.1016/0168-1923(85)90095-4

Tejeda-Sartorius O, Arévalo-Galarza ML. 2012. La floricultura una opción económica rentable para el minifundio mexicano. Agroproductividad 5: 11-27.

The Royal Horticultural Society. [internet]. 2020. Euphorbia fulgens, scarlet plume. [citado 2020 ene 15]. Disponible en: https://www.rhs.org.uk/Plants/24426/ Euphorbia-fulgens/Details

Torres-Navarro H. 2000. Colecta y caracterización de Sprekellia formosissima (L.) Heb., una planta silvestre con potencial ornamental. Tesis de Maestría. Universidad Autónoma Chapingo. Chapingo, México.

Triangle Nursery Ltd. [internet]. 2020. Euphorbia fulgens. [citado 2020 ene 15]. Disponible en: https://www.trianglenursery.co.uk/flower-guides/euphorbia-guide

Van Leeuwen PJ. 1986. Post-harvest treatment of Euphorbia fulgens. Acta Horticulturae 181: 467-471. https://doi. org/10.17660/ActaHortic.1986.181.67

Weslor Flowers Plant Nursery. [internet]. 2020. Scalet Plume-Euphorbia fulgens $125 \mathrm{~mm}$. [citado 2020 ene 15]. Disponible en: https://www.weslorflowers.com/ product/scarlet-plume-euphorbia-fulgens-125mm/ 
Zamudio S. 1995. Las plantas mexicanas del género Pinguicula un grupo de interés hortícola. Revista Chapingo Serie Horticultura 3: 63-69.

Zhang B, Stoltz LP, Snyder JC. 1987. In vitro propagation of Euphorbia fulgens. HortScience 22: 486-488. 